\title{
Genome organisation of Staphylococcus aureus isolates from different populations
}

\author{
W. EL-ADHAMI and P. R. STEWART* \\ Division of Biochemistry and Molecular Biology, Faculty of Science, Australian National University, Canberra \\ ACT 0200, Australia
}

\begin{abstract}
Isolates from three different Staphylococcus aureus populations were examined for restriction fragment length polymorphisms (RFLPs) of total DNA digested with the endonuclease $S m a I$. The populations were: community $S$. aureus isolates collected at random from healthy individuals (38 isolates); methicillin-resistant $S$. aureus (MRSA) type strains involved in separate outbreaks of infection in Melbourne (1982) and Canberra (1990) (two isolates); and a collection of clinical methicillin-sensitive $S$. aureus (MSSA) causing hospital infection (20 isolates). RFLPs with CspI and SmaI and hybridisation analyses of both, showed that the community and the MSSA isolates were not genetically closely related, and, accordingly, they could not be grouped into clusters as seen with the MRSA types. However, a few MSSA isolates were found to be closely related to each other and appeared to be similar to the standard strain $S$. aureus 8325-4 and to some MRSA types. Although there was substantial variability between the three groups, physical mapping with genomic DNA fragments from the standard strain $S$. aureus 8325-4 to probe large fragments generated with $C s p I$ and $S m a I$ from the chromosomes of selected community and MRSA isolates, demonstrated a well conserved genome organisation between representative isolates from the three groups.
\end{abstract}

\section{Introduction}

The population structure of methicillin-multiresistant Staphylococcus aureus (MRSA) and other pathogenic populations of $S$. aureus have been examined by various techniques including enzyme electrophoretic typing [1-3] and restriction fragment length polymorphisms (RFLP) [4, 5]. These analyses revealed that populations appear to be clonal in structure; i.e., these are populations in which the genome appears to be conserved and recombination frequencies are relatively low [6]. This suggests that particular genes and combinations of genes may be selected for in the clinical environment. However, statistical analyses of the extent of clonality within other bacterial populations suggest that the paradigm 'clonality' does not fit all bacterial species. Maynard Smith et al. [7] demon-

Received 2 April 1996; revised version accepted 4 Sept. 1996.

Corresponding author: Dr W. El-Adhami. Present address: School of Biomedical Sciences, Curtin University of Technology, GPO Box U1987, Perth, WA 6001, Australia.

*Present address: CAMBIA, GPO Box 3200, ACT 2601, Australia. strated that bacterial populations can range from almost strictly clonal populations such as Escherichia coli, Salmonella and Haemophilus spp., to the non-clonal fully sexual populations like Neisseria gonorrhoeae.

Recently, it has been demonstrated that exchange of genetic material and recombination events do occur in clonal populations. For example, gene genealogies revealed that horizontal genetic exchange and recombination occur at a significant rate within and between different clonal populations of a bacterial species [816]. Guttman and Dykhuizen [13] concluded that recombination within a clone can be a homogenising force mixing the gene pool, whereas recombination between different clones will act as a diversifying force. Thus, the clonal population can be visualised as sharing a common chromosomal frame, within which individual segments can have independent phylogenetic histories $[6,8,9,11,13]$.

Little information is available about the degree of genetic variability among populations of clinical methicillin-sensitive $S$. aureus (MSSA) and community $S$. aureus. The latter is a population in which selection pressure for virulence and resistance may not occur. This raises the question of whether community and methicillin-sensitive isolates of $S$. aureus resemble 
MRSA isolates in their clonal structure, and whether their genomes are conserved to the extent seen in MRSA populations. An answer to this question may provide a better understanding of the capacity of this bacterium to cause infection or remain commensal. Put another way, is it possible to predict virulence and clinical significance on the basis of knowledge of the presence and arrangements of genes in different staphylococcal lineages, i.e., on purely genetic evidence, without making assumptions about the production of particular enzymes or proteins by individual staphylococcal isolates or clones? Although disease manifestation is the culmination of a complex interaction between the organism, the host and the environment, if genetic or chromosomal correlates of virulence could be identified, the monitoring and surveillance of potential outbreak clones would be possible by relatively simple molecular techniques, e.g., by RFLP analysis of genomic DNA from isolates, or by analysis for particular genes or DNA sequences.

This report describes an attempt to answer these questions by analysing the population structure of a collection of community isolates (collected at random from healthy individuals), a group of clinical MSSA isolates and representative MRSA isolates by RFLP and pulsed-field gel electrophoresis (PFGE) analysis of genomic DNA. The extent of genetic diversity of such populations can be assessed from the extent of polymorphisms of DNA fragments generated by rarecutting restriction endonucleases [4]. The chromosomes of representative isolates were also physically mapped by hybridisation analyses of restriction fragments to examine the extent of conservation of the organisation of chromosomal segements between isolates.

\section{Materials and methods}

\section{Bacterial strains}

S. aureus 8325-4 (ISP8) [17] was kindly provided by Dr P. Pattee, Iowa State University. Strain ANS46 is a methicillin-multi-resistant $S$. aureus (MRSA) extensively studied in this and other laboratories and is a representative of a clonal group referred to as type 46 (and subtypes within), which was dominant in major hospitals in the eastern Australian states between 1982 and 1986 [4, 18-23]. The second Australian MRSA strain is representative of an RFLP type referred to as type I (subtype Ib), prevalent in Canberra hospitals during 1989-1990 [4]. On the basis of previously reported RFLP analyses of Australian MRSA populations (200 isolates examined), the two isolates ANS46 and $\mathrm{Ib}$ were selected to represent the two RFLP types of MRSA that dominated hospitals in major Eastern Australian cities [4,5; unpublished observations]. All clinical MRSA isolates were hospital-acquired and were isolated from patients with systemic infections during outbreaks of MRSA [4]. Clinical MSSA isolates, obtained from the Woden Valley Hospital, Canberra during 1993, were isolated from superficial infections; they express resistance to three or fewer antibiotics of the nine commonly tested for this organism. These isolates were not involved in outbreaks of infection. Community $S$. aureus (CoSA) isolates were collected by nasal swabbing of a random selection of healthy individuals (a random sample of university students), who had no history of hospitalisation, staphylococcal infection or disease in Canberra during 1990; these express resistance to not more than two antibiotics. Isolates were identified as $S$. aureus by growth and mannitol fermentation on Nutrient Agar (Oxoid) with $\mathrm{NaCl} 5 \%$, and by a positive coagulase test and lysostaphin sensitivity. Antibiotic susceptibilities were tested by the BBL Sensi-Disc System by measuring and comparing zones of inhibition of growth around antibiotic impregnated disks to standard resistant and sensitive strains as described by the manufacturer (Becton Dickinson).

\section{DNA preparation}

Growth and preparation of cells and digestion of their genomic DNA with rare-cutting endonucleases in agarose blocks before pulsed-field electrophoresis have been described previously $[22,24]$. The $S$. aureus genome contains approximately $35 \mathrm{~mol} \% \mathrm{G}+\mathrm{C}$, and accordingly restriction endonucleases that recognise GC-rich sequences cleave the genome relatively infrequently to produce a small number of fragments. Sma I (5'-CCCGGG-3') and CspI (5'-CGGA/TCCG-3') have proved useful in the physical mapping of strain 8325 [25]; therefore, these were used in the present study. Plasmid DNA was prepared by the alkaline-lysis method as described elsewhere [26] with the addition of a digestion step with lysozyme $4 \mathrm{mg} / \mathrm{ml}$ and lysostaphin $1 \mu \mathrm{g} / \mathrm{ml}$.

\section{Pulsed-field gel electrophoresis}

Clamped homogeneous electric field (CHEF DRII; BioRad) gel electrophoresis was performed in agarose $1 \%$ gels at $200 \mathrm{~V}$ for a total of $26 \mathrm{~h}$ at $12-14^{\circ} \mathrm{C}$ with Tris-borate buffer $(45 \mathrm{~mm}$ Tris, $45 \mathrm{mM}$ boric acid and $1 \mathrm{mM}$ EDTA, $\mathrm{pH}$ 8.3) with the following pulse parameters: first set, $23 \mathrm{~s}$ for $4 \mathrm{~h}$; second set, ramped with initial forward time $1 \mathrm{~s}$, final forward time $40 \mathrm{~s}$ for $22 \mathrm{~h}$. The same electrophoresis parameters were used to separate DNA fragments generated by digestion with SmaI, Csp I and SacII endonucleases. After electrophoresis, gels were stained with ethidium bromide $0.5 \mu \mathrm{g} / \mathrm{ml}$, destained in water, and photographed over a UV transilluminator. Southern blotting and probing of CHEF filters was as described previously [22] except that the probes used were SmaI genomic fragments resolved by $\mathrm{CHEF}$ electrophoresis in high purity low melting temperature agarose $1 \%$ (SeaPlaque; FMC). The bands were excised from the gel and radiolabelled 
in the agarose by the Megaprime DNA labelling system [27-28].

\section{Analysis of DNA fragment patterns on PFGE gels}

DNA fragment analysis was performed as described previously $[4,5]$. Values of $p$, a measure of the genetic distance approximating the fraction of nucleotides different in the genomes examined, and the genetic events that alter the structure of chromosomes, were calculated as described previously [29-30]. For the purpose of this study, a type is used to describe closely related RFLP patterns generated from genomic DNA prepared from the isolates with rare-cutting endonucleases (e.g., SmaI). Each RFLP type is subdivided into subtypes, which are separated by $p$ values of 0.003-0.008; within each subtype the isolates share identical SmaI (or other endonuclease) fragment patterns and have a $p$ value of zero [4]. A group is used to describe a number of closely related individual RFLP types (also referred to as a clone), which are separated by a genetic distance of 0.015 [4].

\section{Physical mapping}

The strategy for physically mapping the chromosomes involved digesting the genomes of the selected strains with rare-cutting endonucleases and resolving the fragments generated by PFGE. The alphabetic nomenclature (and numbering) of fragments followed that of Pattee [25] where the largest fragment was first (see Table 1), proceeding in a descending order with reduced size. The ordering of fragments and construction of a circular map was achieved with data derived from hybridisation of individually labelled SmaI fragments from strain ISP8 (8325-4) with the genomic fragments from the selected strains generated by different endonucleases (SmaI, Csp I and $S a c \mathrm{II}$ ). Briefly, labelled SmaI fragments from ISP8 were hybridised to transfer-filters of CHEF-separated fragments of genomic DNA from ANS46, Ib, Co11 and Co14 (ISP8 was included each time as a reference), digested with CspI or SmaI. A single ISP8 SmaI fragment should hybridise to itself in a Sma I digest of ISP8, and should hybridise to one or more fragments obtained with the other endonuclease. This provides the overlap required for ordering of the fragments and comparison with the SmaI and Csp I physical map of ISP8 [25,31]. One matter to be taken into account when comparing the hybridisation patterns is that ISP8 is a derivative (8325-4) of strain NCTC 8325 in which fragment SmaI-F has been lost (due to the loss of phages $\phi 11$ and $\phi 13$ carried on fragment SmaI-F), to be replaced by a fragment $c .73 \mathrm{~kb}$ smaller (Fig. 1, compare the brightness of band SmaI-H to SmaI-G in ISP8), which co-migrates with fragment SmaI-H [17].

Table 1. Restriction fragments obtained with SmaI-digested DNA from clinical and community isolates and separated by PFGE

\begin{tabular}{|c|c|c|c|c|c|}
\hline & \multicolumn{5}{|c|}{ Isolates* } \\
\hline & ANS46 & ISP8 & $\mathrm{I}(\mathrm{b})$ & Col1 & Col4 \\
\hline & $\mathrm{a}^{\dagger}>650$ & $\mathrm{~A}^{\dagger} \leqslant 650$ & $\mathrm{a}^{\prime \dagger}>650$ & $1^{\dagger} \geqslant 700(2)$ & $1^{\prime \dagger} \geqslant 700$ \\
\hline & b 410 & B 380 & $b^{\prime} 452.5$ & 2332 & $2^{\prime} 475$ \\
\hline & c 370 & C 350 & $c^{\prime} 370$ & 3272 & $3^{\prime} 317$ \\
\hline & d 325 & D 300 & $d^{\prime} 335$ & 4175 & $4^{\prime} 300$ \\
\hline & e 300 & E 285 & $e^{\prime} 300$ & 5155 & $5^{\prime} 245$ \\
\hline & f 153 & G 187.5 & X 157.5 & 6132 & $6^{\prime} 190(2)$ \\
\hline & g 145 & H 153 (2) & $f^{\prime} 153(2)$ & 7117 & $7^{\prime} 175$ \\
\hline & h 128 (2) & I 128 & $\mathrm{~h}^{\prime} 128$ & $897(2)$ & $8^{\prime} 132$ \\
\hline & i 110 & J 87 & $i^{\prime} 110(2)$ & 970 & $9^{\prime} 112$ \\
\hline & j 87 & K 62 & $j^{\prime} 87$ & 1055 & $10^{\prime} \quad 60$ \\
\hline & $\mathrm{k}_{1} 75$ & L 53 & $\mathrm{k}_{1}^{\prime} 75$ & 1147 & $11^{\prime} 55$ \\
\hline & $\mathrm{k}_{2} 62$ & M 47 & $\mathrm{k}_{2}^{\prime} 62$ & 1240 & $12^{\prime} 27$ \\
\hline & $1_{1} 53$ & N 36 & $l_{1} 53$ & $1336(2)$ & $13^{\prime} \dagger \leqslant 5$ \\
\hline & $\mathrm{l}_{2} 47$ & O 31 (2) & $\mathrm{l}_{2}^{\prime} 47$ & 1427 & \\
\hline & m 36 & P 24 & $\mathrm{~m}^{\prime} 36$ & 1524 & \\
\hline & n 31 (2) & Q 9 & $\mathrm{n}^{\prime} 31$ & 169 & \\
\hline & o 24 & & $o^{\prime} 24$ & & \\
\hline & p 21 & & $\mathrm{p}^{\prime} 21$ & & \\
\hline & q 9 & & $\mathrm{q}^{\prime} 9$ & & \\
\hline $\begin{array}{l}\text { Aggregated } \\
\text { size }\end{array}$ & $3195 \mathrm{~kb}$ & $2966.5 \mathrm{~kb}$ & $3395 \mathrm{~kb}$ & $3121 \mathrm{~kb}$ & $2983 \mathrm{~kb}$ \\
\hline
\end{tabular}

Numbers in parentheses indicate the apparent number of coinciding fragments that co-migrate in single bands. The alphabetic nomenclature of fragments shown in this table (described in text) is used for constructing the physical maps. Fragment sizes are defined in $\mathrm{kb}$. Nomenclature of isolates is as described in text.

*ANS46 and Ib, methicillin-multi-resistant isolates (MRSA); ISP8, clinical methicillin-sensitive isolate (MSSA); Col1 and Co14, community methicillin-sensitive isolates.

$\dagger \leqslant, \geqslant$, indicate that sizes in this range are only approximations; with the PFGE conditions used it was not possible to determine the sizes more accurately. The molecular size was determined with $\lambda$ phage concatemers, $\lambda$ phage DNA digested with HindIII, and ANS46 SmaI fragments as standards. 


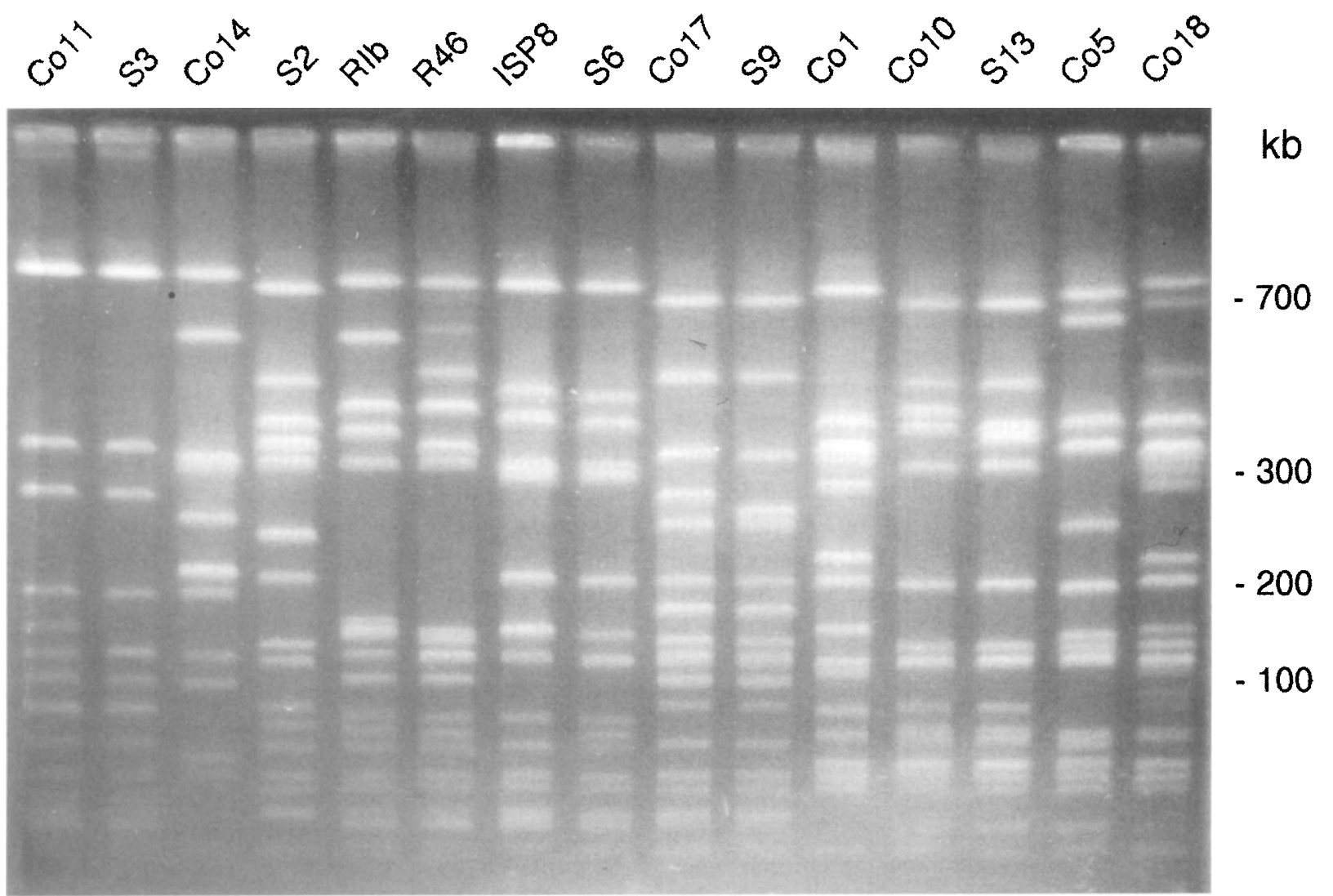

Fig. 1. PFGE separation of SmaI-digested DNA from a selection of $S$. aureus isolates. Co-, community isolates; S-, clinical methicillin-sensitive (MSSA) isolates; R-, clinical methicillin-resistant (MRSA) isolates; R46, ANS46; RIb, type $\mathrm{Ib}$ (see text for further details). Size markers derived from $\lambda$ phage markers and ANS46 fragments [22] are indicated on the right of the figure.

\section{Results}

\section{Antibiotic susceptibilities}

ANS46 and Ib (type Ib) were resistant to penicillin, methicillin, tetracycline, chloramphenicol, gentamicin, erythromycin and kanamycin. Clinical MSSA isolates were resistant to three or fewer antibiotics, including penicillin, tetracycline and erythromycin. Other than penicillin, the community isolates were sensitive to all antibiotics tested. Two exceptions were seen with isolates Col4 and Co17; the former was resistant to erythromycin and tetracycline, the latter was resistant to erythromycin.

\section{PFGE of DNA from isolates digested with CspI and SmaI}

Digestion with SmaI of total cellular DNA and PFGE revealed $15-21$ bands of $10-700 \mathrm{~kb}$ in the eight community isolates, the MSSA isolates and the representative MRSA types tested. Fig. 1 compares the SmaI restriction patterns for the community isolates and the clinical MSSA isolates with ISP8 (MSSA), and ANS46 and Ib (MRSA). The MRSA strains appear to be related with an estimated $p$ value of 0.012; the MRSA strains (ANS46 and Ib) are more closely related to each other than either is to ISP8. In previous studies, Inglis et al. [22] demonstrated that upon the loss of the mec region (a cluster of resistance genes c. $40 \mathrm{~kb}$ in length) from the chromosome of ANS46, the PFGE patterns in the deletants appeared to be more related to ISP8 than did the parental strain ANS46. Although a few community and MSSA isolates showed closely related fragmentation patterns indicating a close genetic relationship with a $p$ value of 0.002 (e.g., compare SmaI patterns of the isolates in Fig. 1, lanes 1 and 2), the extent of genomic variation within each group (with $p$ values as high as 0.14 ) did not permit their division into clusters as seen with MRSA isolates $[4,5]$. These results were also true of a larger group of 30 community and $20 \mathrm{MSSA}$ isolates examined by RFLP (data not shown). Plasmid DNA, when present, would be visualised as extra fragments. However, estimates of genetic distances would be minimally affected, as no more than two plasmids per isolate were identified. Table 1 shows the range of size of genomic fragments, and their multiplicity, generated by $\mathrm{Sma}$ I for selected isolates.

\section{Physical mapping by ordering of fragments generated by restriction endonucleases}

Hybridisation analysis was carried out with individual Sma I fragments from the mapped strain ISP8 as 
labelled probes. These probes were hybridised to Southern filters of Sma I-digested genomic DNA from the community isolates, and from ISP8 and ANS46. Fig. 2 shows a schematic representation illustrating the results obtained from hybridisation with the ISP8 Sma I fragment probes. Each labelled fragment from ISP8 hybridised to a fragment of the same relative position of migration (i.e., starting from the largest size fragment and proceeding to the smallest) in the digestion patterns of the other isolates with some exceptions. For example, fragment SmaI-A of ISP8 hybridised to a fragment of similar size, and to the largest, in all other isolates (Fig. 2). Although some differences were seen between the hybridisation patterns (e.g., the community hybridisation patterns obtained with fragment SmaI-J of ISP8 were different from that of ISP8, ANS46 and Ib; Fig. 2), the data suggested that the physical (restriction site) maps for the isolates were generally similar and, hence, physical mapping of the genomes of selected isolates was carried out. ISP8 was selected as representative of clinical MSSA isolates. The community isolate Coll was selected as representative of CoSA isolates which might be related to MSSA types (Fig. 1), and the other community isolate (Col4) was selected because it appeared to be distantly related to all other isolates.

Given the close similarity between the chromosomes of the three clinical strains (evident from restriction fragments) and between the community and the MSSA and ISP8 (evident from hybridisation analysis), hybridisation of SmaI fragments from ISP8 to fragments in CspI and SmaI digests of ANS46, Ib, Col1, and Co14 should permit the construction of a physical map for the four isolates. Fig. 3 shows an example of the hybridisation analysis used for physical mapping, whereby fragment SmaI-G from ISP8 was used as a probe. The complexity of Sac II digestion profiles (particularly the co-migration of fragments in some gels, data not shown) did not allow an unambiguous assignment of all SacII sites in the SmaI or CspI fragments. However, it was possible to identify a partial map for the SacII sites (shown for the clinical strains). Fig. 4 compares the physical maps for SmaI, CspI and the partial map of SacII sites for these isolates.

The use of ISP8 SmaI fragments as probes allowed the determination of the physical location of the Sma I-P and Csp I-J fragments of ISP8, which were not reported by Pattee, who also reported the map location of fragment SmaI-N to be between SmaI-A and -B [25]. However, the hybridisation analysis described here indicates that fragment $S m a \mathrm{I}-\mathrm{M}$ is actually in this position. Also, SmaI-N and -O were found to be adjacent, and were located between SmaI-G and -C. The exact order cannot be decided unequivocally because SmaI-N and -O could not be resolved adequately on preparative gels and, accordingly, these fragments had to be used as a mixed probe. An extra fragment (SmaI-Q) was identified which is adjacent to Sma I-P, most probably between SmaI-E and -P (Fig. 4). Fragments SmaI-C and SmaI-H of ISP8 were found to hybridise to an identical Sac II fragment, and thus must be contiguous. This was also found with

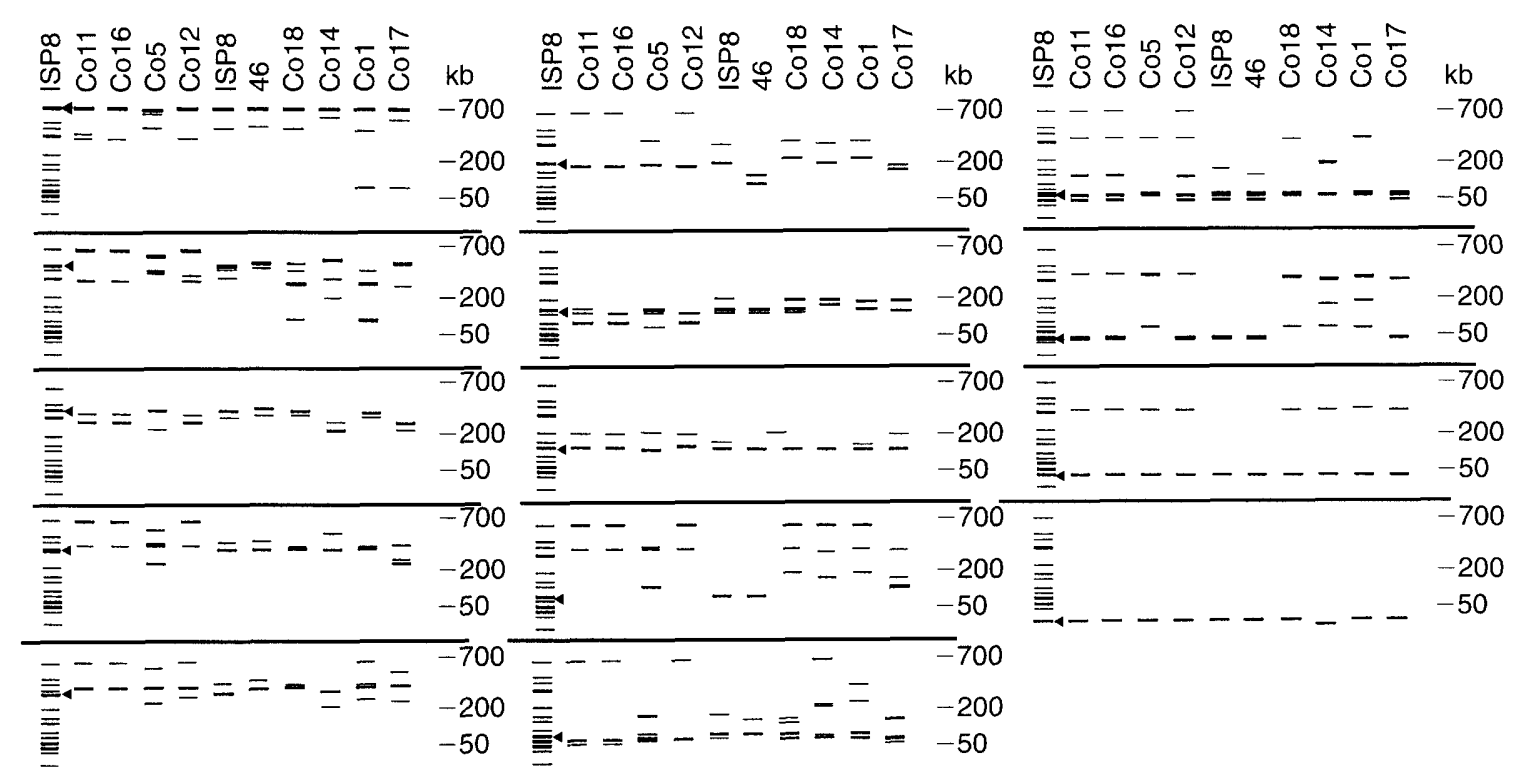

Fig. 2. Schematic representation of the hybridisation patterns obtained for SmaI-digested DNA from ANS46 and the community $S$. aureus (Co-) isolates with radiolabelled SmaI-generated fragments (indicated by 4) from ISP8 genomic DNA. SmaI fragmentation pattern for ISP8 is shown on the left of each panel of the diagram, whereby individual Sma I fragments used separately each time are indicated by bold face and marked with ( 4 ) in descending order. The resultant major hybridising fragments from the SmaI-digested genomes of the different isolates are shown in bold (fragments shown in plain line in the community isolates and ANS46 represent secondary hybridising fragments of weaker intensity). Size markers are indicated on the right of the figure. 

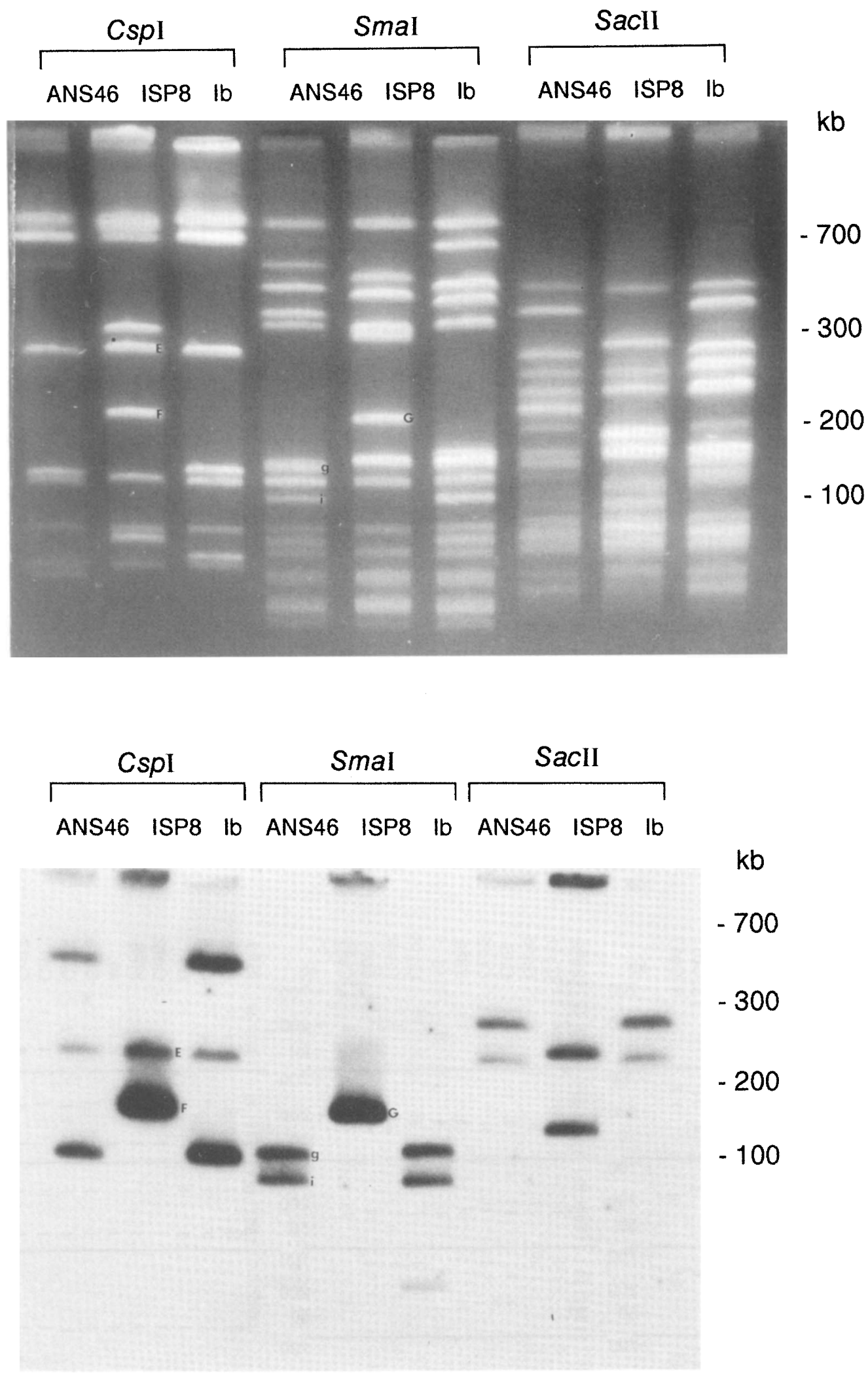

Fig. 3. PFGE of CspI; SmaI and SacII-digested DNA from the three clinical isolates (ANS46, ISP8 and Ib), and an autoradiograph showing hybridisation with fragment SmaI-G from ISP8. This figure is an example of the strategy used for physical mapping of the genomes from the different isolates (see text for further details). SmaI fragments from ISP8 (and their equivalent from $C s p$ I) are indicated by upper-case letters. ANS46 and Ib Sma I and CspI fragments hybridising with SmaI-G from ISP8 are indicated by lower-case letters (e.g., ANS46 SmaI-g and i). A size marker is also indicated. Differences in residual DNA in the loading wells were commonly seen in PFGE hybridisations. Electrophoresis conditions were as used for Fig. 1. 


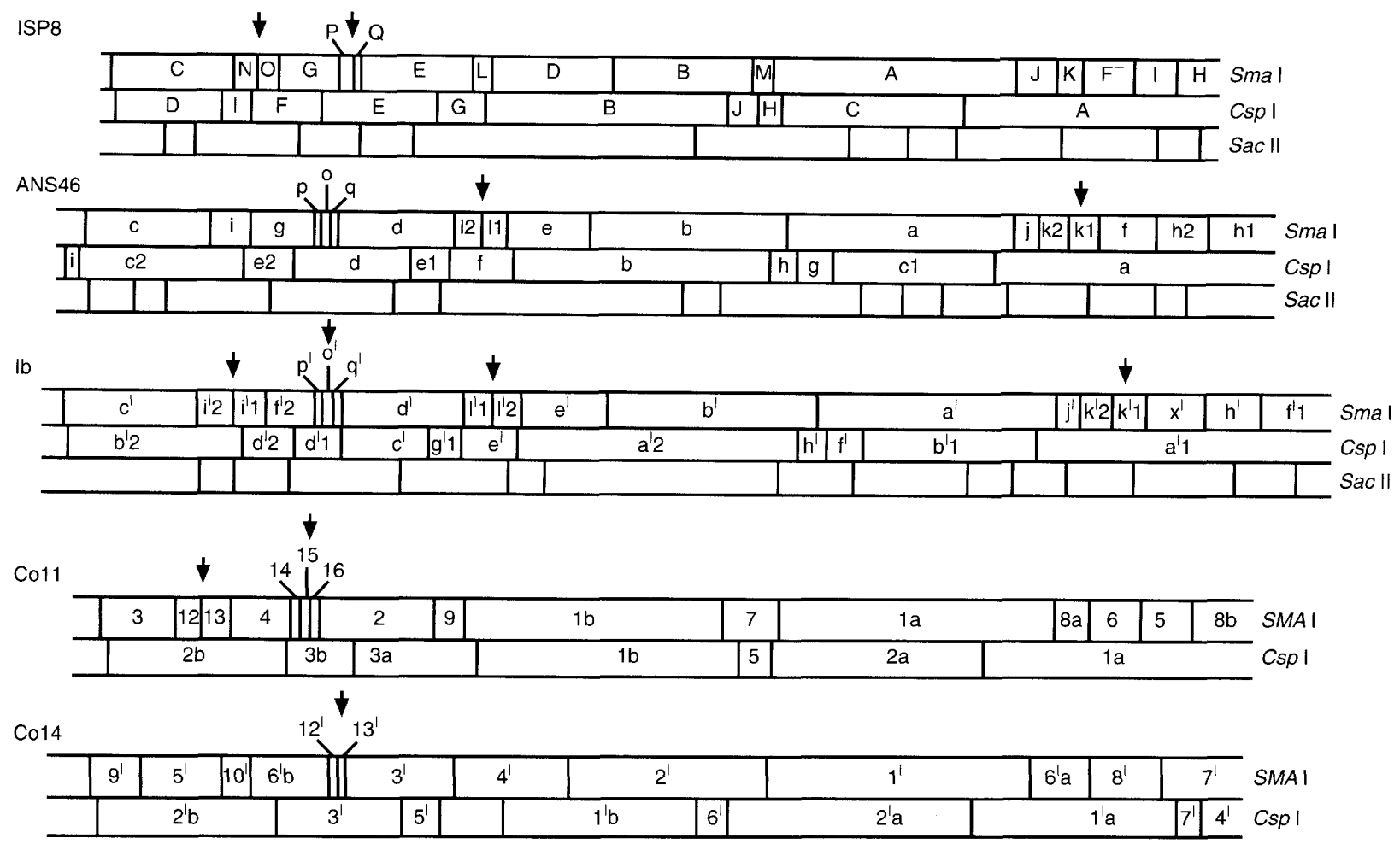

Fig. 4. Physical maps of clinical methicillin-sensitive ISP8, clinical methicillin-resistant isolates ANS46 and Ib, and community S. aureus (Co11 and Col4) isolates. The maps show SmaI and CspI sites for all isolates examined and a partial map of SacII sites for the clinical isolates (see text). Marked $(\downarrow)$ fragments refer to equivocal determination of positive with regard to other fragments. Circularity of the chromosomes from community isolates has not been determined. Fragment nomenclature is as described in Materials and methods.

both ANS46 and Ib (i.e., SmaI-c and -h are contiguous). Sac II was not used in the mapping of the CoSA isolates, and hence it is not possible to confirm the circularity of their physical maps, although they show a similar chromosomal organisation to the MSSA and MRSA isolates.

\section{Discussion}

The clonal nature of MRSA isolates that dominate hospital environments in Australia $[4,5]$ poses some important questions about the nature of staphylococcal populations beyond the clinical or hospital environment. For example, are those staphylococci associated with healthy individuals as part of their normal flora (i.e., commensal) also clonal, and if so, are the clones different or similar to those which constitute MRSAs? Another question is whether community isolates have the same virulence potential as MRSAs.

The analysis of RFLPs described here shows that the community and MSSA isolates were not closely related with pair-wise differences in $p$ values as high as 0.142 when compared to ISP8. A large number of genetic events is necessary to account for the high $p$ values measured within and between the community and the MSSA isolates [4,5]. Nonetheless, a few of these isolates showed low values of $p$ when compared with each other; for example Col7 and S9 in Fig. 1 differed by a $p$ value of 0.003 . It is possible that the clinical MSSA isolates have been community-acquired, which would provide an explanation for the few similarities identified between the two groups. However, the route of transmission of isolates between the community and the clinical environment cannot be determined due to the lack of sufficient epidemiological history. The standard reference strain ISP8, although originally isolated in the 1950s in England, appears to be similar to recent MRSAs and to some recent MSSAs. Two MSSA isolates were in fact identical to ISP8 on the basis of SmaI-generated genome fragments. The $p$ value difference between ISP8 and the resistant strains was 0.03 .

It is noteworthy that the MRSA isolates were from systemic infections and were involved in outbreaks of infection, and have dominated Eastern Australian hospitals for more than a decade [4,5], whereas the clinical MSSA isolates were from minor infections and were not linked to any outbreaks. Whether dominant clonal MRSA populations could be attributed to selection pressures exerted by antibiotic usage, or are the result of other selection forces remains to be determined. As closely related MRSA and MSSA types co-exist in the clinical environment [5], this suggests that other selection forces are at play in the clinical environment. 
Although the analyses described here suggest that particular clonal populations appear to be dominant and more successful in the clinical environment, the lack of extensive epidemiological data does not permit further conclusions about the relationship between virulence and clonality. The answers to such a correlation might be partly explained by examining regions of the chromosome which appear to be absent from the non-clonal populations.

The physical maps presented here, although not complete in some instances, permit certain conclusions about the genome organisation of independently isolated $S$. aureus types. The differences between ISP8, and ANS46 and Ib (Fig. 4) appear to be related to the presence of additional DNA sequences, seen as larger sized or extra fragments, and thus, overall, indicate a greater size of the genome in ANS46 and Ib. The presence of extra DNA in the MRSA isolates might be explained by the presence of plasmids, bacteriophages and transposable elements, chromosomally integrated [6] or existing extra-chromosomally. In the latter case, the extra fragments would be seen among the smaller bands, because plasmids and phages generally do not exceed $c .50 \mathrm{~kb}$ in staphylococci [32].

Several examples illustrate the differences between ISP8 and ANS46 fragment sizes. Fragment SmaI-D of ISP8 was found to hybridise to SmaI-e of ANS46 and Sma I-E hybridised to SmaI-d in ANS46 (and in Ib); from this their relative physical position on the map was determined (Fig. 4). Pattee et al. [33] have demonstrated that in an 8325 derivative carrying Tn916 (encodes tetracycline resistance; integration site $\Omega 1105$ mapped to SmaI-E) and the plasmid pLI475 [34], fragment SmaI-E migrates more slowly than SmaI-D as a result of the increase in size. This provides a simple explanation for the difference in the relative physical position on the map of the two fragments from ANS46 (and in Ib), assuming a similar insertion has occurred in their genomes. Other, more complicated, explanations may be possible. It is noteworthy that fragment SmaI-G of ISP8 (which carries the insertion point for the mec region) hybridised to two fragments in SmaI digests of both ANS46 and Ib, but only to one fragment in the community isolates. Inglis et al. [22] have demonstrated that the loss of c. $40 \mathrm{~kb}$ of DNA spanning the mec region (containing a Sma I site) in ANS46 leads to the loss of two fragments (SmaI-g and $-\mathrm{i}$ in their nomenclature, 145 and $110 \mathrm{~kb}$ ) to be replaced by a new fragment which migrates at about the same position of fragment SmaI-G of ISP8. The mec gene itself (when present) has been genetically mapped to fragment Sma I-G [35] in ISP8. Because the mec region contains a Sma I site [22], the hybridisation of fragment Sma I-G from ISP8 is to two fragments (SmaI-g and -i) in strains (ANS46, Ib) carrying the mec region. ANS46 and $\mathrm{Ib}$ also contain a fragment of $c .75 \mathrm{~kb}$ in size
(Sma I-k 1 , Fig. 1). Sma I-k 1 did not hybridise with any ISP8 Sma I fragments, and its size is almost identical to the size of DNA deleted from ISP8 fragment SmaI-F ( $\phi 11$ and $\phi 13$ account for $73 \mathrm{~kb}$ ) [17]. Indeed, this fragment was found to be homologous to fragment SmaI-F (obtained from NCTC 8325, data not shown), indicating an extra SmaI site in the ANS46 and Ib chromosomal region equivalent to SmaI-F of NCTC 8325 (parental strain of ISP8). The order of SmaI and Csp I fragments from both Col1 and Col4 genomes is similar, if not identical, to that of ISP8. Although the differences between ISP8 and the MRSA types could be attributed to the presence of additional DNA in the chromosomes of the resistant types, the differences between ISP8 and the community isolates appear to be of a different nature. Because the genomes of ISP8 and the community isolates are almost identical in size, mutational events ranging from single nucleotide substitutions (e.g., ISP8 fragments SmaI-J and -K were found to be part of fragment Sma I-1 in both Col1 and Co14, indicating the absence of two SmaI sites, potentially a consequence of point mutations) to rearrangements such as inversions, transpositions and duplications are required to explain the polymorphisms observed $[6,36]$. This certainly does not exclude the possibility that insertions or deletions have occurred, although the two types of events would need to be balanced to maintain the genome size approximately constant.

The physical mapping method described can be complicated by several factors, the most important of which is the presence of repeated sequences in the genome [6]. The analyses reported here appear to be complicated by the presence of some repeated DNA sequences. Background hybridisation was observed with some fragments of the digested DNA (this was particularly noticeable when the larger SmaI fragments were used as probes). ISP8 does not carry the repeated sequences carried by ANS46 (such as TN554 and IS257) [20,37-40]. However, this does not exclude the presence of the other repeated DNA, e.g., rRNA genes $[33,41]$.

RFLP analysis has provided important information on the stability versus the flexibility of the bacterial genome. Recently, Weinstock [36] described the variability in RFLP patterns of five mutant strains of Escherichia coli $\mathrm{K}-12$. He concluded that despite this variability, the basic $E$. coli map can be applicable to other strains, taking into consideration the sort of genetic events discussed above. The analyses described here for $S$. aureus indicate that, like $E$. coli, the picture seen with clinical versus community strains of $S$. aureus is one in which the stability of the overall chromosome structure is maintained, yet a significant degree of variability occurs between (and within) the two populations. The genetic properties of MRSA isolates are becoming more variable, as is shown by the differences between the RFLP patterns. Although 
genetic exchange and recombination would introduce polymorphic differences into the recipient population, these patterns would only be found in the regions after recombination occurred; the rest of the genome would display a clonal relationship. Chromosome organisation appears to be well conserved in several other bacterial species [42]. It would be of interest to examine the genetic relatedness and the chromosomal organisation of international MRSA isolates and compare these to Australian isolates. Physical mapping studies of the mec region from ANS46 and from North American MRSA isolates indicate that they share some similarities [23].

Further studies are currently in progress to examine the presence of conserved DNA sequences characteristic of clonal and possibly virulent $S$. aureus strains, which might carry virulence genes, that are absent in the non-clonal community strains. It will be of interest to compare $S$. aureus with the genomes from other staphylococcal species, and to examine the gene order and general organisation of the genomes between $S$. aureus and the coagulase-negative staphylococci.

\section{References}

1. Musser JM, Schlievert PM, Chow AW et al. A single clone of Staphylococcus aureus causes the majority of cases of toxic shock syndrome. Proc Natl Acad Sci USA 1990; 87: 225-229.

2. Musser JM, Selander RK. Genetic analysis of natural populations of Staphylococcus aureus. In: Novick RP (ed) Molecular biology of the Staphylococci. New York, VCH Publishers. 1990: 59-67.

3. Musser JM, Kapur V. Clonal analysis of methicillin-resistant Staphylococcus aureus strains from intercontinental sources: association of the mec gene with divergent phylogenetic lineages implies dissemination by horizontal transfer and recombination. J Clin Microbiol 1992; 30: 2058-2063.

4. El-Adhami W, Roberts L, Vickery A, Inglis B, Gibbs A, Stewart PR. Epidemiological analysis of a methicillin-resistant Staphylococcus aureus outbreak using restriction fragment length polymorphisms of genomic DNA. J Gen Microbiol 1991; 137: 2713-2720.

5. Stewart PR, El-Adhami W, Inglis B, Franklin JC. Analysis of an outbreak of variably methicillin-resistant Staphylococcus aureus with chromosomal RFLPs and mec region probes. $J$ Med Microbiol 1993; 38: 270-277.

6. Krawiec S, Riley M. Organization of the bacterial chromosome. Microbiol Rev 1990; 54: 502-539.

7. Maynard Smith J, Smith NH, O'Rourke M, Spratt BG. How clonal are bacteria? Proc Natl Acad Sci USA 1993; 90: 4384-4388.

8. Milkman R, Bridges MM. Molecular evolution of the Escherichia coli chromosome. III. Clonal frames. Genetics 1990; 126: 505-517.

9. Maynard Smith J. The evolution of prokaryotes: does sex matter? Ann Rev Ecol System 1990; 21: 1-12.

10. Dykhuizen DE, Green L. Recombination in Escherichia coli and the definition of biological species. J Bacteriol 1991; 173: 7257-7268.

11. Maynard Smith J, Dowson CG, Spratt BG. Localized sex in bacteria. Nature 1991; 349: 29-31.

12. Guttman DS, Dykhuizen DE. Detecting selective sweeps in naturally occurring Escherichia coli. Genetics 1994; 138: 993-1003.

13. Guttman DS, Dykhuizen DE. Clonal divergence in Escherichia coli as a result of recombination, not mutation. Science 1994; 266: $1380-1383$

14. Whatmore AM, Kapur V, Sullivan DJ, Musser JM, Kehoe MA. Non-congruent relationships between variation in $\mathrm{emm}$ gene sequences and the population genetic structure of group $\mathrm{A}$ streptococci. Mol Microbiol 1994; 14: 619-631.

15. Whatmore AM, Kapur V, Musser JM, Kehoe MA. Molecular population genetic analysis of the enn subdivision of group A streptococcal emm-like genes: horizontal gene transfer and restricted variation among enn genes. Mol Microbiol 1995; 15: 1039-1048.

16. Reda KB, Kapur V, Goela D, Lamphear JG, Musser JM, Rich RR. Phylogenetic distribution of streptococcal superantigen $S S A$ allelic variants provides evidence for horizontal transfer of SSA within Streptococcus pyogenes. Infect Immun 1996; 64: 1161-1165.

17. Patel AH, Foster TJ, Pattee PA. Physical and genetic mapping of the protein A gene in the chromosome of Staphylococcus aureus 8325-4. J Gen Microbiol 1989; 135: 1799-1807.

18. Heneine N, Stewart PR. Physiological determination of methicillin resistance in Staphylococcus aureus: comparison of clinical and genetically derived isolates. $J$ Antimicrob Chemother 1986; 17: 705-715.

19. Gillespie MT, Lyon BR, Loo LSL, Matthews PR, Stewart PR, Skurray RA. Homologous direct repeat sequences associated with mercury, methicillin, tetracycline and trimethoprim resistance determinants in Staphylococcus aureus. FEMS Microbiol Lett 1987; 43: 165-171.

20. Matthews PR, Reed KC, Stewart PR. The cloning of chromosomal DNA associated with methicillin and other resistances in Staphylococcus aureus. J Gen Microbiol 1987; 133: 1919-1929.

21. Skinner S, Inglis B, Matthews PR, Stewart PR. Mercury and tetracycline resistance genes and flanking repeats associated with methicillin resistance on the chromosome of Staphylococcus aureus. Mol Microbiol 1988; 2: 289-292.

22. Inglis B, Matthews PR, Stewart PR. Induced deletions within a cluster of resistance genes in the mec region of the chromosome of Staphylococcus aureus. J Gen Microbiol 1990; 136: 2231-2239.

23. Dubin DT, Chikramane SG, Inglis B, Matthews PR, Stewart PR. Physical mapping of the mec region of an Australian methicillin-resistant Staphylococcus aureus lineage and a closely related American strain. J Gen Microbiol 1992; 138: $169-180$

24. Stewart PR, El-Adhami W, Inglis B. Pulsed-field gel electrophoresis: applications to bacteria. In: Dale JW, Sanders PG Methods in gene technology. London, JAI Press. 1994: 177-205.

25. Pattee P. The genetic map of Staphylococcus aureus. In: Sonenshein AL, Hoch JA, Losick R (eds) Bacillus subtilis and other gram-positive bacteria: biochemistry, physiology, and molecular genetics. Washington, DC, American Society for Microbiology. 1993: 489-496.

26. Sambrook J, Fritsch EF, Maniatis T (eds). Molecular cloning. A laboratory manual. Cold Spring Harbor, NY, Cold Spring Harbor Laboratory Press. 1989

27. Feinberg AP, Vogelstein B. A technique for radiolabeling DNA restriction endonuclease fragments to high specific activity. Anal Biochem 1983; 132: 6-13.

28. Feinberg AP, Vogelstein B. A technique for radiolabeling DNA restriction endonuclease fragments to high specific activity. 'Addendum' Anal Biochem 1984; 137: 266-267.

29. Upholt WB. Estimation of DNA sequence divergence from comparison of restriction endonuclease digests. Nucleic Acids Res 1977; 4: 1257-1265.

30. Nei M, Li W-H. Mathematical model for studying genetic variation in terms of restriction endonucleases. Proc Natl Acad Sci USA 1979; 76: 5269-5273.

31. Pattee PA. Genetic and physical mapping of the chromosome of Staphylococcus aureus NCTC 8325. In: Drlica K, Riley M (eds) The bacterial chromosome. Washington, DC, American Society for Microbiology. 1990: 163-169.

32. Lyon BR, Skurray RA. Antimicrobial resistance of Staphylococcus aureus: genetic basis. Microbiol Rev 1987; 51: 88-134.

33. Pattee PA, Lee HC, Bannantine JP. Genetic and physical mapping of the chromosome of Staphylococcus aureus. In: Novick RP (ed) Molecular biology of the staphylococci. New York, VCH Publishers. 1990: 41-58.

34. Clewell DB, Flannagan SE, Ike Y, Jones JM, Gawron-Burke C. Sequence analysis of termini of conjugative transposon $\mathrm{Tn}$ 916. J Bacteriol 1988; 170: 3046-3052.

35. Kuhl SA, Pattee PA, Baldwin JN. Chromosomal map location 
of the methicillin resistance determinant in Staphylococcus aureus. J Bacteriol 1978; 135: 460-465.

36. Weinstock GM. Bacterial genomes: mapping and stability. ASM News 1994; 60: 73-78.

37. Stewart PR, Dubin DT, Chikramane SG, Inglis B, Matthews PR, Poston SM. IS257 and small plasmid insertions in the mec region of the chromosome of Staphylococcus aureus. Plasmid 1994; 31: 12-20.

38. Byrne ME, Gillespie MT, Skurray RA. 4', 4" adenyltransferase activity on conjugative plasmids isolated from Staphylococcus aureus is encoded on an integrated copy of pUB110. Plasmid 1991; 25: 70-75.
39. Dubin DT. Tn554 and related DNA: mapping and tracking the staphylococci chromosome. In: Novick RP (ed) Molecular biology of the staphylococci. New York, VCH Publishers. 1990: $85-98$.

40. Matthews PR, Inglis B, Stewart PR. Clustering of resistance genes in the mec region of the chromosomes of Staphylococcus aureus. In: Novick RP (ed) Molecular biology of the staphylococci. New York, VCH Publishers. 1990: 69-83.

42. Green CJ, Vold BS. Staphylococcus caureus has clustered tRNA genes. J Bacteriol 1993; 175: 5091--5096.

42. Cole ST, Girons IS. Bacterial genomics. FEMS Microbiol Rev 1994; 14: $139-160$ 